\title{
Insights into Chinese prostate cancer with RNA-seq
}

\author{
Anirban Sahu ${ }^{1}$, Matthew K Iyer ${ }^{1}$, Arul M Chinnaiyan ${ }^{1,2,3,4,5}$ \\ ${ }^{I}$ Michigan Center for Translational Pathology; ${ }^{2}$ Department of Pathology; ${ }^{3}$ Department of Urology; ${ }^{4}$ Comprehensive Cancer Cen- \\ ter; ${ }^{5}$ Howard Hughes Medical Institute, University of Michigan Medical School, 1400 E. Medical Center Dr, 5316 Cancer Center, \\ Ann Arbor, MI 48109-0602, USA
}

Cell Research (2012) 22:786-788. doi:10.1038/cr.2012.50; published online 27 March 2012

The prevalence of certain driver mutations in prostate cancer varies across ethnic populations. A recent study uses RNA-seq to profile the mutational landscape of prostate cancer in a Chinese population to gain additional insights on ethnic variation in this disease.

Prostate cancer remains a leading cause of cancer morbidity and mortality in men, accounting for approximately one million new cases and 260000 deaths per year worldwide [1]. Incidence and mortality rates vary widely across geographic regions and ethnic groups. In particular, Asian populations have a substantially lower incidence rate than Caucasians or African Americans. In 2010, 1/10 000 Chinese men received a diagnosis of prostate cancer compared to 15/10 000 Caucasian-Americans, 23/10 000 African-Americans, and 9/10 000 Asian-Americans [2]. Although differences in cancer screening practices may account for some of these differences, understanding the underlying genetic variation affecting disease pathogenesis remains an important priority.

The clinical presentation of prostate cancer is widely heterogeneous. Many patients survive for decades with slowgrowing disease while others progress

Correspondence: Arul M Chinnaiyan Tel: +1-734-615-4062; Fax: +1-734-615-4498

E-mail: arul@umich.edu quickly to metastasis. In recent decades, genomic profiling of this disease has uncovered recurrent genetic alterations driving progression such as deletion of the PTEN tumor suppressor, arm-level amplification of chromosome 8q, and amplification of androgen receptor (AR) in recurrent disease [3]. One area of emphasis has been the androgen family of steroid hormones that stimulate growth of the prostate gland by binding to and activating ARs, which in turn translocate to the nucleus and function as a transcription factor. Prostate cancer cells also depend on androgen signaling for survival, and the clinical management of this disease includes androgen deprivation as a front-line therapy. In 2005, a game-changing link was established between the androgen signaling pathway and prostate cancer through the discovery of a genetic rearrangement that causes the fusion of two independent genes on chromosome 21: the androgen-regulated gene TMPRSS2 and the ETS transcription factor ERG, a previously known oncogene in sarcoma [4]. The TMPRSS2-ERG gene fusion has been causally linked to cancer progression by promoting invasion in vitro, and overexpression of the fusion product in mice shows greatly enhanced prostate cancer development [5].

The majority of prostate cancer research has studied Caucasian populations. While genetic events such as TMPRSS2-ERG fusion and PTEN loss can be found in other populations, recent studies show that the prevalence of major driver genes varies greatly across people of different ethnic backgrounds. For example, the prevalence of the TMPRSS2-ERG gene fusion is higher in Caucasians $(\sim 50 \%)$ than African Americans $(\sim 31 \%)$ or Asians (16\%$30 \%)[6,7]$. Additionally, PTEN loss occurs more frequently in Caucasian (up to $40 \%$ ) than Asian (15\%) populations [8]. These discrepancies indicate that ethnic bias in molecular profiling may neglect important genetic factors affecting prostate cancer pathogenesis across the globe. Supporting this notion, genome-wide association studies focusing on Asian and African-American populations uncovered different risk loci from similar studies of Caucasians $[9,10]$.

Tumor profiling studies have been advanced by the recent maturation of high-throughput sequencing technologies, which enable more unbiased analysis of DNA, RNA, and epigenetic modifications. In particular, transcriptome sequencing (RNA-seq) represents an attractive and information-rich modality as it captures data useful for discovering gene fusions, identifying single nucleotide variants and small insertions/ deletions, quantifying gene expression, and assessing the prevalence of alternatively spliced isoforms and previously unreported transcripts (see Figure 1) $[11,12]$. In their recent study of prostate 

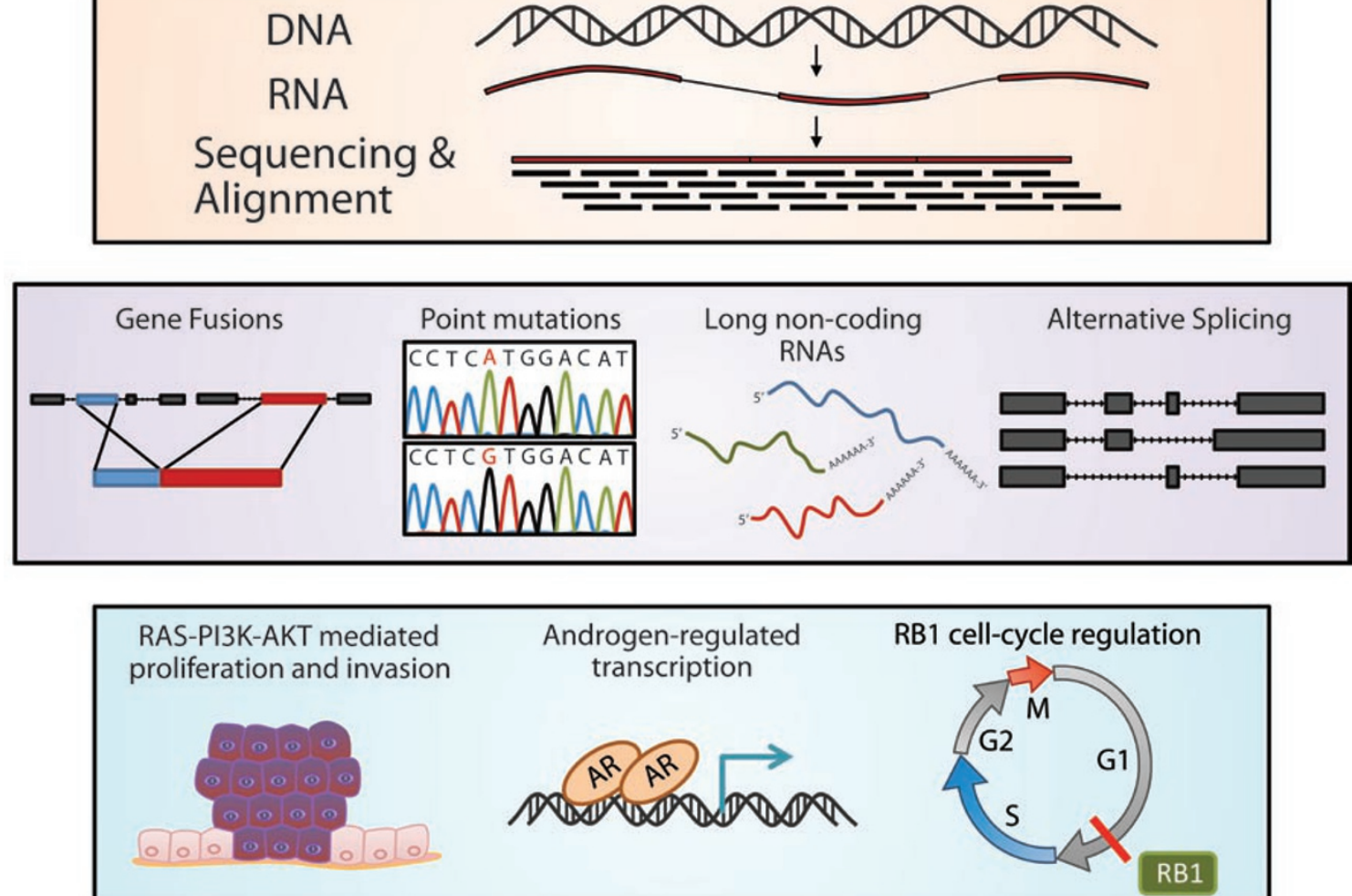

Figure 1 Schematic overview of the study. RNA-seq performed on 14 patient tumor and adjacent normal tissues identified gene fusions, point mutations, long non-coding RNAs, and alternatively spliced transcripts. Analysis of the aggregated mutational profiles implicated Ras-PI3K-AKT signaling, androgen signaling, and RB1 cell cycle regulation as major pathways affecting pathogenesis of prostate cancer in this Chinese patient cohort.

cancer in Chinese patients, Ren and colleagues [13] used RNA-seq to profile genetic aberrations in 14 patient tumor versus adjacent normal tissues and confirmed their findings in an independent cohort of 54 patient tumor samples. Their results provided a global view of the transcriptome, identified tumor-associated gene fusions and somatic single nucleotide mutations, and monitored the expression of long non-coding RNAs and alternatively spliced genes. The study uncovered three recurrent gene fusions, including the TMPRSS2-ERG fusion, a previously unreported event involving the adjacent genes USP9Y and TTY15 on chromosome Y, and an interchromosomal translocation involving genes CTAGE5 and KHDRBS3. The prevalence of TMPRSS2-ERG gene fusion was $19.1 \%$ (3/14 in RNA-seq cohort, 10/54 in validation cohort), which is consistent with previous studies in Asian populations. Additionally, the authors identified 309 point mutations located in coding regions of genes. None of the point mutations were recurrent at a single genomic position, but 3 of the 14 samples harbored mutations in the gene UTP $14 C$. Finally, the authors observed the expression of long noncoding RNAs and alternatively spliced transcripts. They reported 137 differentially expressed long non-coding RNAs and several isoforms with intron retention or exon skipping.

Comparison of the aggregated mutational profiles from all patients across a database of known signaling networks implicated three major pathways in this prostate cancer cohort: Ras-PI3KAKT signaling, androgen signaling, and RB1 cell cycle regulation. Ongoing research efforts should prioritize the synthesis and integration of these results with other complementary studies. For example, a recent study of prostate cancer in Chinese men by Chen et al. [14] used DNA-based single nucleotide polymorphism (SNP) arrays to discover molecular aberrations and proposed that deregulation of cell cycle machinery may account for differences in pathogenesis between Western and Asian populations. Also, this new data could be compared with other prostate tumor RNA-seq datasets to determine whether any of the aberrations found by the authors are enriched in individuals of Chinese descent [15].

Although RNA-seq can be an effective genomic assay, its shortcomings should not be overlooked. Variant calling can be challenging or impossible for genes with low expression levels; in these cases, DNA-based sequenc- 
ing methods would be more sensitive. Furthermore, editing of RNA transcripts by enzymes such as APOBEC3G complicates somatic variant calling for A-to-G nucleotide changes. Moreover, RNA-seq cannot detect focal DNAlevel deletions and amplifications. Given the relevance of PTEN and other tumor suppressors frequently deleted in prostate cancer, a complementary assay such as targeted exome sequencing would add important insight into the comprehensive landscape of prostate cancer mutations. Also, gene fusions nominated by RNA-seq must be secondarily validated to determine whether the fused gene arose from a somatic chromosomal rearrangement. In many cases, such as the previously reported SLC45A3-ELK4 fusion transcript in prostate cancer, "read-through" fusion transcripts can arise independently of genomic alterations [16]. Interestingly, the fusion genes USP9Y and TTTY15 reported by Ren and colleagues [13] are separated by just 9 kilobases on chromosome $\mathrm{Y}$ and lie on the same genomic strand. Further characterization of the USP9Y-TTTY15 fusion product and its role in prostate cancer pathogenesis will be informative

Furthermore, this study highlights the importance of being cognizant of ethnic differences while exploring molecular mechanisms of disease. The signaling networks implicated by this study suggest that prostate cancer progression depends on a consistent set of cellular processes, but the genetic differences among ethnic groups may correspond to different molecular mechanisms for deregulating these processes. Overall, this study provides a valuable dataset, confirms substantial variation in the prevalence of certain genetic aberrations, and nominates new genes that may contribute to cancer pathogenesis in the Chinese population. Most importantly, this study reminds us to acknowledge and appreciate genetic diversity in human disease. Ultimately we hope that expanding our knowledge about genetic differences among ethnic groups will improve medical decision making in clinical oncology.

\section{References}

1 Jemal A, Bray F, Center MM, et al. Global cancer statistics. $C A$ Cancer $J$ Clin 2011; 61:69-90.

2 Shao Q, Ouyang J, Fan Y, et al. Prostate cancer in the senior men from rural areas in east district of China: Contemporary management and 5-year outcomes at multi-institutional collaboration. Cancer Lett 2012; 315:170-177.

3 Squire JA, Park PC, Yoshimoto M, et al. Prostate cancer as a model system for genetic diversity in tumors. $A d v$ Cancer Res 2011; 112:183-216.

4 Tomlins SA, Rhodes DR, Perner S, et al. Recurrent fusion of TMPRSS2 and ETS transcription factor genes in prostate cancer. Science 2005; 310:644648.

5 Tomlins SA, Laxman B, Varambally S, et al. Role of the TMPRSS2-ERG gene fusion in prostate cancer. Neoplasia 2008; 10:177-188.

6 Magi-Galluzzi C, Tsusuki T, Elson P, et al. TMPRSS2-ERG gene fusion prevalence and class are significantly different in prostate cancer of Caucasian, African-American and Japanese patients. Prostate 2011; 71:489-497.

7 Lee K, Chae JY, Kwak C, et al. TMPRSS2-ERG gene fusion and clinicopathologic characteristics of Korean prostate cancer patients. Urology 2010; 76:1268.e7-e13.

8 Mao X, Yu Y, Boyd LK, et al. Distinct genomic alterations in prostate cancers in Chinese and Western populations suggest alternative pathways of prostate carcinogenesis. Cancer Res 2010; 70:5207-5212.

9 Liu F, Hsing AW, Wang X, et al. Systematic confirmation study of reported prostate cancer risk-associated single nucleotide polymorphisms in Chinese men. Cancer Sci 2011; 102:19161920.

10 Wang M, Liu F, Hsing AW, et al. Replication and cumulative effects of GWAS-identified genetic variations for prostate cancer in Asians: a case-control study in the ChinaPCa consortium. Carcinogenesis 2012; 33:356-360.

11 Prensner JR, Iyer MK, Balbin OA, et al. Transcriptome sequencing across a prostate cancer cohort identifies PCAT1, an unannotated lincRNA implicated in disease progression. Nat Biotchnol 2011; 29:742-749.

12 Wang Z, Gerstein M, Snyder M. RNASeq: A revolutionary tool for transcriptomics. Nat Rev Genet 2009; 10:57-63.

13 Ren S, Peng Z, Mao JH, et al. RNAseq analysis of prostate cancer in the Chinese population identifies recurrent gene fusions, cancer-associated long noncoding RNAs and aberrant alternative splicing. Cell Res 2012; 22:806-821.

14 Chen JH, He HC, Jiang FN, et al. Analysis of the specific pathways and networks of prostate cancer for gene expression profiles in the Chinese population. Med Oncol 2011 Oct 30 doi:10.1007/s12032-011-0088-5

15 Pflueger D, Terry S, Sboner A, et al. Discovery of non-ETS gene fusions in human prostate cancer using next-generation RNA sequencing. Genome Res 2011; 21:56-67.

16 Maher CA, Kumar-Sinha C, Cao X, et al. Transcriptome sequencing to detect gene fusions in cancer. Nature 2009; 458:97-101. 